\title{
ZUR \\ GESCHICHTE DES OTFRIDISCHEN VERSES IM ENGLISCHEN.
}

(Fortsetzung von Anglia VIII, Anz. s. 49 ff.)

IV.

Die siebentreffige laugzeile nach Orm bis in das

15. jahrhundert.

Beim Ormulum konnte man im zweifel sein, ob man es mit einer sieben- oder achttreffigen langzeile $z u$ tun habe. Für das erstere sprach der umstand, dass nie schwerere ableitungssilben den schluss bildeten, für das letztere der umstand, dass die vorletzte silbe immer lang war. Das erste denkmal, wo ohne zweifel nur sieben hebungen beabsichtigt sind, wo die entwickelung des kurzen Otfridischen metrums durch die achttreffige langzeile hindurch zur siebentreffigen zur vollendeten tatsache geworden ist, ist The Passion of our Lord.' Schipper sagt, in nachahmung des originals in franzọsischen alexandrinern sei der alexandriner vorwiegend, indess kämen auch andere versarten, z. b. septenare vor, aber die alte langzeile fehle gan\%. Trantmann, Wissnıann und Einenkel treten dieser ansicht entgegen. Trautmann behauptet: 'Alle ersten halbzcilen haben vier hebungen, bei der zweiten halbzeile finden sich sehr viele mit drei hebungen; wir haben also sieben hebungen, die keinen alexandriner geben'.

Dies ist richtig. Unter den ersten hundert versen zählte ich achtundsechzig, die ohne zweifel siebentreffig sind, wo bei nngezwnngener, natiirlicher betonung sieben hebungen herauskommen; der rest von zweiunddreissig versen konnte allerdings

1 Ms. Jes. Coll. 0xf. I, Arch. I, 29; gedruckt bei Morris, An 0ld English Misccllany, London 157\%. 
alexandrinisch gelesen werden, wenn man keine senkung fehlen liess. Da aber das fehlen von senkungen in diesem denkmal wider ganz gewöhnlich, anch von Schipper zugegeben ist, und sich auch im zweiten halbverse findet, der im allgemeinen regelmässiger gebant ist als der erste, so müssen wir entschieden diese freiheit auch für einen teil jener ubrig gebliebenen 32 verse gelten lassen. Es lassen sich dann noch 14 verse ohne muthe siebentreffig lesen und nur 18 sechstreffige, sogenannte alexandriner bleiben. Hierzu gehören v. 11:

prýtty wintre and móre / he wés amóng Monkúnne, der aber vielleicht richtiger so zu lesen ist:

prýtty wíntre and móre he wés $\|$ amóng Mónkúnne;

ferner v. 14:

He wes swype of-longed $\|$ to his fader blysse.

Ferner v. 24, 26, 28, 32, 33, 40, 42, 45, 50, 51, 66, 76, 81, 87, 91, 100 .

Von diesen ersten 100 versen haben noch 73 weiblichen schluss und nur 27 männlichen. Wir sehen also zwar einen fortschritt gegentuber dem Ormulum, wo alle verse weiblich endeten, andererseits aber doch noch ein festhalten an dem hergebrachten und einfluss. der ursprtinglich achthebigen form des metrums. Der ausgang des ersten halbverses ist bald männlich, bald weiblich, nur die alexandrinischen verse haben auffallenderweise immer weiblichen ausgang. Sollte hierin doch noch ein rest der alten tonfähigkeit der letzten silbe zu sehen sein? Im allgemeinen bleiben die nebensilben allerdings ohne treff in unserem denkmale, und die schwachen flexionssilben sogar immer. Das gesetz der treffigung der letzten, weiblichen silbe nach langer stammsilbe ist aufgehoben. Nur schwere endsilben, wie -yng(e), -inde, nesse $u$. a. sind noch hebungsfähig, z. b. érndýnge (: brynge), lésýnge, mótýnge, preching (:king), wenynge (: pinge), pruwinge; rydinde, syngynde; gladnesse, sopnesse (: lesse); warlyche; erest; Kayphas (: was), Jesus a. a.

Zusammengesetzte wörter können naturlich ebenfalls auf jeden bestandteil einen treff nehmen: goddspélle, lyfdaye, chepmen, wimmen, wymmon, leofmon etc.

Der auftakt zu anfang fehlt in der halfte der veree; einige male steht doppelter auftakt. Nach der cäsur ist der auftakt meist da; auch hier steht bisweilen doppelter. Gewöhnlich sind je zwei langzeilen durch endreim gebunden, einige male aber 
vier bis sechs, so v. 29-32 mete : ete : hete : speke; v. $65-68$ suneday : gray : palefray : may; v. 89-94 ihere : supere : chere : were: yvere : nere; ferner v. $173-76,177-80,185-88,189-92$, 393-96, 689-92 etc. Die reime sind im allgemeinen rein. Dass bisweilen schwere endsilben im reime stehen, sahen wir oben; einige male beschränkt sich sogar der reim auf schwache endsilben: blessy (: bethany), sone (: to me), redi (: by), vondy (:very); jedenfalls ist dies wider ein rest der alten weise.

Auf demselben standpunkte steht unser metrum in A lutel soth Sermun.' Bei Morris ist es in kurzzeilen gedruckt, von denen je die zweite und vierte reimen, die erste und dritte reimlos sind. Im ganzen sind 46 langzeilen und 8 viermal gehobene kurzzeilen. Die letzteren, sämmtlich weiblich endigend, durchbrechen nach v. 16 bei Morris die einheitliche form des gedichtes und reimen paarweise. Von den 46 langzeilen haben 34 im ersten halbvers 4 hebungen, die zweiten halbverse haben sämmtlich 3 hebungen. Wir haben also 34 regelmässige langzeilen und nur 12 'alexandriner', von denen sich aber noch einige bei auslassung von senkungen, schwebender betonung, septenarisch lesen liessen. Der erste halbvers dieser letzteren 12 verse. endet wider immer weiblich, also genau wie in der Passion.

Die nebensilben erhalten auch hier nicht mebr den treff, ausgenommen einige schwerere und in zusammensetzungen: léchürs, cheping, horlyngs; chápmén, monquelle.

Der auftakt zu anfang fẹlt in $2 / 3$ der verse; nach der cäsur dagegen ist das umgekehrte verhältniss, $d . h$. er steht in $2 / 3$ der verse. In v. 42 (bei Morris) steht doppelter auftakt nach der cäsur: heore bred and heore ale.

Hieran schliesst sich Whon holy chireche is under vote. ${ }^{2}$ Es sind regelrechte siebentreffige langzeilen, ausgenommen v. 19 und v. 29 , wo nur 6 hebungen vorhanden sind, allerdings wider bei weiblichem ausgang des ersten halbverses. In den anderen versen endet der erste halbvers bald männlich, bald weiblich, der zweite halbvers dagegen, mit ansnahme der ersten 6 verse, immer weiblich.

1 Jes. Coll. Oxf. M. 29 und ms. Cott. Calig. A IX; gedruckt bei Morris An Old English Miscellany s. 157 .

2 Morris, a. a. o. s. 89. 
Von fällen, wo schwere endungen treffigung zulassen, seien genannt: wúrpsipe, würshipe, Sýmón, Clémént; vielleicht ist hierher auch die endung -es des plurals zu zählen in bispes and clerekes, knyhtes, kynges, yefles. Genaueres freilich lässt sich nicht bestimmen. Wir stehen hier in dieser zeit des schwankens auf unsicherem boden. Es ist ganz der willktur des verfassers anheimgestellt, welche endsilben er fur schwer genug halten will, einen treff $\mathrm{zu}$ tragen.

Fehlen des auftaktes ist wider ganz gewöhnlich.

Die ersten sechs verse, die einzigen männlichen, reimen unter einander, dann reimen je vier, mit ausnahme der beiden reimpaare $v .29 / 30$ und $31 / 32$.

$\mathrm{Zu}$ nennen ist hier ferner no. 5 bei Morris: Fortune, ein kleines fragment, bestehend aus vier unter einander gereimten siebentreffigen langzeilen.

Zwei andere hierhergehörige gedichte sind: On serving Christ und The Annunciation of the Virgin Mary. ${ }^{1}$ Das erstere, aus 78 versen bestehend, ist sehr holprig gebaut. Sehr viele senkungen fehlen; ebenso fehlt der anftakt in der mehrzahl der verse, wenigstens zu anfang. Aber das metrum der siebentreffigen langzeile ist trotz der vielen abweichenden verse deutlich erkennbar: erster halbvers 4 hebungen, zweiter halbvers 3. -

Der zweite halbvers endet ebenso wie der erste bald männlich, bald weiblich. Also der unterschied im ausgange der beiden halbverse, der in der Passion noch sehr anffallend war, ist ganz aufgegeben, so dass jetzt nichts mehr an die ehemalige achtmal gehobene form erinnert.

Die verse sind nicht paarweise durch reim gebunden, sondern, ähnlich wie in den tiraden der altfranzösischen Chansons de geste, geht derselbe reim durch eine unbestimmte anzahl von versen hindurch, meist durch $6-8$, aber auch durch 10 , einmal sogar durch 14 . -

Das zweite gedicht besteht aus 18 versen, von denen zwei unvollständig sind, und ist fast noch schlechter gebaut als das erste. Trotzdem die mehrzahl der verse nur 6 hebungen zu haben scheinen, sind wol auch hier siebentreffige langzeilen beabsichtigt. Diejenigen ersten halbverse, die nur 3 hebungen

1 Ms. Jes. Coll. 29; beide gedruckt bei Morris, a. a. o. s. 99 u. 100. 
haben, enden immer weiblich. Die verse sind paarweise gereimt. Der auftakt fehlt meist, selbst nach der cäsur.

Die treffigung ist in beiden denkmälern unsicher, daher auch der charakter der einzelnen verse. Es lässt sich gar nicht feststellen, ob ein vers mit 6 oder 7 hebungen zu lesen sei. Sicher ist nur die hebungsfihigkeit schwerer endsilben und des zweiten bestandteiles von zusammensetzungen, so in On serving Christ: grónínge, ridynde, schynynde; drerlyke, sunfulle; likwise; loverd, fulluhı, monkunnes; in The Annunciation: Archaungle, nomon.

Wir kommen nun zu jenen denkmälern, die Schipper als die eigentlichen repräsentanten der 'gemischten literatur' hinstellt, wo er eine mischung von alexandrinern und septenaren sieht. Es sind dies zunächst eine grosse anzahl von legenden und legendenartigen erzählungen, besonders aus dem suden; dann die chronik des Robert of Gloucester und schliesslich ein Fragment of popular Science.

Die legenden sind uns in zablreichen, von circa 1300 bis in das 16. jahrhundert reichenden handschriften erhalten. Die ältesten geordneten texte finden sich in den manuskripten Harl. 2277 und Ashmol. 43.1 Abweichend davon sind die legenden behandelt im ms. Laud. 108, wo oft das versmaass erweitert, und im ms. Egert. 1993, wo der text vereinfacht ist. Das ms. Vernon kombiniert die älteren versionen. Spätere handschriften sind: ms. Cott. Jul. D. IX, eine abschrift von Ashmol. 43; ferner ms. Addition. 10301 im Brit. Museum und ms. Addition. 10626. An ms. Harl. 2277 schliesst sich an das ms. Corp. Chri. Coll. Cambr. 145. Aus dem 15. jahrhundert stammen die mss. Bodl. 779, L,ambeth 223 and Trin. Coll. Cambr. R. 3, 25. Wichtig ist ferner das ms. S. John's Coll. Cambr. B. 6 in nördlicherem dialekt. Teile der suldlichen sammlung sind enthalten in den mss. Tanner Oxf. 17, King's Coll. Cambr. 15 und Philips 8253 (in Cheltenham). ${ }^{2}$

1 Vgl. Horstmann, Altengl. Jegenden, Paderborn 1875 und Altengl. Legenden, neue folge, Heilbronn 1881.

2 Von ausgaben und sammlungen von legenden sind besonders die folgenden zu nennen: Horstmann, Altenglische Legenden, Paderborn 1875; Ders., Altengl. Legenden, Heilbronn 1878: Ders.. Altengl. Legenden, neue folge, Heilbronn 1881; Ders., Leben Jesu, Münster 1873. Furnivall, Early English poems and lives of Saints, Berlin 1862 (Philological Society). - Small, English metrical Homilies, Edinburgh 1862. 
Zum zwecke der untersuchung greifen wir aus den verschiedenen mss. einige der wichtigsten legenden heraus.

Aus ms. Harl. 2277 nehmen wir die legenden Thomas Becket, St. Dunstan, St. Christopher und St. Margaret.' Der vers ist bei allen vieren unverkennbar der siebentreffige. Verse von nur sechs hebungen durften in den bei Mätzner abgedruckten stticken kaum zu finden sein, abgesehen von v. 8 in St. Dunstan, der auch bei Schipper als beweis fur die mischung angefthrt wird:

pat folc stod in gret wónder $\|$ and álso in gréte dónte.

Die verse sind auffallend regelmässig, besonders wenn wir sie mit denen der eben betrachteten denkmäler vergleichen. Nur in St. Margaret sind sie weniger glatt. Fehlen des auftaktes ist auch in Thomas Becket, St. Dunstan and St. Christopher noch ganz gewőhnlich: in 50 versen $11-16 \mathrm{mal}$; seltener ist fehlen der senkung im innern. Ebenso finden sich mehrfache senkung und mehrfacher auftakt, besonders in St. Margaret, wo öfters dreisilbiger anftakt steht, und schwere silben im innern za verschleifen sind. Ein unterschied im schluss des ersten and zweiten halbverses ist nicht mehr zu finden; sie enden beide nach belieben männlich oder weiblich.

In der treffigung stimmen alle vier uberein: nur zusammengesetzte wörter und solche mit schwerer endsilbe lassen schwebende betonung $\mathrm{zu}$. Darauf grtinden sich reime wie mansinge (: kinge T. B. 1874), (: bringe 1901), nothing (: king 2035), woninge (: bringe St. Cr. 75), tipinge (: bringe St. M. 245). Auch im innern finden sich nur derartige worter mit schwebender betonung: Herston (St. D. 24), seopinge (St. M. 247), glowinge (214); ferner godnisse, clennisse, schindisse; manqueller. Beispiele fur den gebrauch derartiger wörter im innern mit nur einer hebung sind aus St. Cr.: hondred (143), clanliche (65), wymen (180); aus St. M.: schyndful (126).

Gewőhnlich sind je zwei langzeilen durch endreim zu einem reimpare verbunden, öfters zeigen auch vier verse denselben reim, in St. Margaret sogar sechs: sende : wende : hende : ende : wende : sende.

1 Alle vier, wenigstens teilweise, abgedruckt bei Mätzner, Altengl. Sprachproben. Die legende von Thomas Becket wurde ansserdem herausgegeben von Black (London 1845), sie umfasst 2508 verse und ist die 'perle der ganzen sammlung'. 
Eine andere version von St. Margaret, ganz in strophen von vier zeilen (aaa), findet sich im ms. Auchinl. fol. $16^{\mathbf{b}}$ aus dem anfang des 14. jahrhunderts. ${ }^{1}$ Hier finden sich auffallend viele sechsheber. Während der zweite halbvers immer drei hebungen enthält, zeigt $1 / 3$ der ersten halbverse statt vier auch nur drei; meist endet dann allerdings der erste halbvers weiblich. Das verhältniss ist folgendes: von 100 ersten halbversen sind 31 dreihebig weiblich, 7 dreihebig männlich, 21 vierhebig weiblich, 41 vierhebig männlich. Im übrigen sind die verse glatt gebaut; mehr als die hälfte zeigen auftakt; doppelter auftakt ist selten, fehlen von senkungen nur ganz ausnahmsweise zu finden.

In bezug auf die treffigung können wir keinen sicheren schluss ziehen. Nur einmal steht das part. sturbling im reime (v. 233), aber selbst hier haben wir nicht schwebende betonung, sondern nur einen fall jener beim Ormulum erwähnten tonumsetzung, wie sie sich noch in späterer zeit gerade beim part. präs. öfters findet. Im innern tragen oft genug wörter wie wimen, sinful nur ein en treff. Trotzdem sind wol einige fälle schwebender betonung für unser denkmal zuzugeben in wörtern wie leman (92), Mergrele, fairnesse (48), tiding (24), bigining (70), vielleicht auch in noping (282), maiden, angel. Schwächere endsilben sind tonlos, denn sie finden sich ofters auch in den viertreffigen weiblichen halbversen als uberzählige silben.

Dieselbe version in strophen von vier zeilen (aaaa) findet sich unter dem titel Meidan Maregrete im ms. Trin. Coll. Cambr., gedruckt bei Cockayne im 13. bande der E. E. T. S. Auch hier zeigen die ersten halbverse oft nur drei hebungen statt vier. Schwebende betonung ist nur noch in wörtern wie feirnesse, wisdom, slourbing (: spring), loverd, meidan zulässig; sonst zählt eine weibliche silbe auch am ende des halbverses nicht mehr.

Aus ms. Ashmol. 43 mögen die legenden Geburt Jesu, Fegefeuer des heil. Patrick und St. Juliana als beispiele dienen. ${ }^{2}$ Auch hier haben wir sehr regelmässige siebentreffige

1 Gedruckt bei Horstmann, Altengl. Legenden, neue folge, 1881 und bei Hickes, Thesaurus I, 224 (vgl. Guest, History of Engl. Rhythms).

2 Die ersten beiden sind gedruckt bei Horstmann, Altengl. Legenden, Paderborn 1875, die letztere bei Cockayne, E. E. T. S. 1872. 
langzeilen und nur äusserst selten statt ihrer verse von sechs hebungen. Im bau unterscheiden sie sich nicht von denen des ms. Egert. 1993. Wăhrend fehlen des auftaktes häufig vorkommt, stehen die senkungen im innern fast ganz regelmässig; wo sie feblen, ist es gewöhnlich zwischen zwei wörtern; schwebende betonung zweier anf einander folgender silben desselben wortes im innern der verse ist sehr selten; aus der Geb. Jes. kann ich nur childhód anfuhren, aus St. Patr. Irlond (v. 8), aus St. Jul. gar keinen fall. Auch am ende des verses wird sie immer seltener und selbst wörter wie womman (Geb. Jes. 218), lemmon, cheping (St. Jul. 130) fangen an, nur eine hebung zu tragen. Doppelter auftakt und doppelte senkung werden noch oft angetroffen.

Neben dem endreime, der je zwei langzeilen verbindet, steht in der Geburt Jesu von v. 1-80 noch binnenreim. Die reime sind im allgemeinen sehr genau.

Das ms. Laud. 108 der Bodley'schen bibliothek in Oxford (vgl. Horstmann, Leben Jesu, Münster 1873, einleitung) enthält ca. 60 legenden, von denen wir das Leben Jesu und Seinte Marie Maudeleyn' als repräsentanten wählen wollen. Die von Horstmann erwähnten erweiterungen der verse bestehen hauptsächlich in therladung des auftaktes und der senkungen; ferner zeigt die zweite vershälfte bisweilen vier hebungen, so dass wir achttreffige verse haben. Diese letzteren falle sind , besonders zahlreich zu anfang der Magdal, so gleich in v. 2: \| wíse and unwíse, zóngue and olde.

Bisweilen haben wir auch siebenheber im verhältniss von $3: 4$, statt des ublichen von $4: 3$, indem die cassur, statt nach der vierten, schon nach der dritten hebung fallt. Fehlen des auftaktes oder der senkung ist selten.

Je zwei langzeilen sind wider dnrch endreim gebunden: In Maudeleyn kommen daneben viele binnenreime vor. Im Leben Jesu sind die endung -ynye und andere schwere endsilben oft trägerinnen des reimes: likynge (: singe 82), offringe (: bringe 447), falshede (: sede 214, 509), godhede (: sede), womman (: bigan 787); ein beispiel fur reimende franzossische endungen bietet lechour: tresour (136). - Andere proben aus dem ms. Land. 108, so aus dem prolog, ans Fabian, ans dem inhalt

- Gedruckt bei Horstmann, Altengl. Legenden, Heilbronn 1878. 
der sammlung und aus Julian sind zu finden bei Horstmann in der einleitung zu seiner ausgabe und in dem Leben Jesu s. 5. Auch hier sind uberall viele verschleifungen erforderlich.

Im gegensatz zu ms. Laud. 108 verkurzt nach Horstmann das ms. Egert. 1993 gern die legenden und sucht sie in eine knappere form zu bringen. Man vergleiche dafur die beiden versionen der Geburt Jesu nach ms. Ashmol. 43 und nach ms. Egert. 1993, beide abgedruckt bei Horstmann, Altengl. Legenden 1875. Die wenigen verse aus dem prolog (no. 3) und aus dem Adventgedicht (no.1), abgedruckt bei Horstmann a. a. 0 . in der einleitung, bestätigen dies ebenfalls. Der auftakt und die senkungen sind weniger tuberladen, die zahl der hebungen ist auf 7 oder gar nur 6 beschränkt; fehlen der senknngen im innern findet wider häufiger statt, sowol zwischen zwei wörtern, wie zwischen zwei silben desselben wortes.

Alle diese drei versionen, repräsentiert durch 1) die mss. Ashmol. 43 und Harl. 2277, 2) das ms. Land. 108 und 3) das ms. Egert. 1993, sind benutzt im ms. Vernon. Die therarbeitung ist nach Horstmann leicht zu erkennen, und besonders sind die ans Laud. 108 entnommenen sttlcke vereinfacht worden. In den bei Horstmann gegebenen proben aus dem Adventgedicht und aus $0 f$ the Feste of pe Concepcion of vre Lady finden wir statt der langen verse des ms. Laud. wider knappe siebentreffige langzeilen, darunter sogar einige sechstreffige. Schwebende betonung scheint besonders im letzteren wider häufiger beabsichtigt zu sein.

Die letzte grosse legendensammlung ist im ms. Bodl.779. Aus ihr ist Barlaam and Josaphat bei Horstmann, Altengl. Legenden 1875, gedruckt. Auch hier sind die verse sehr knapp, so dass manche wider nur 6 hebungen statt 7 zeigen; so unter den ersten 50 versen circa 10. Daneben fehlt der anftakt zu anfang oder nach der cäsur in der hälfte der verse; anch im innern vermisst man oft senkungen, und schwebende betonung tritt ein, wie die reime zeigen: comyng (: ping 94), ginnyng (: king 151), glading (: ping 201), hontyng (: gronyng 98); goodnesse (: rychesse 357), (: schennesse 955),

$955 \|$ and léve in góodnésse

956 \| and deize in schénnésse,

kingdom (: nom 1187); auch quickliche (v. 1107) erfordert wol zwei hebungen. - Eine andere probe aus diesem ms. ist die 
bei Horstmann, einleitung, abgedruekte ans The Gospel of Seint John (no. 7).

Aus ms. Trin. Coll. Cambr. B. 14, 39 (13. jahrh.) ist bei Wright, Reliqu. antiquae s. 144 ein Judasfragment von 33 versen gedruckt, wo die verse mit 6 hebungen an zahl sogar die mit 7 hebungen tubertreffen. Im tubrigen sind bau und treffigung dieselben wie in den anderen legenden.

Bei Wtulker, Altengl. Lesebuch stehen zwei legenden aus Furnivall, Early English poems and lives of Saints. Es sind dies die legenden St. Katherine und Jadas Iscariot, wo sich thberall 7 hebungen finden bei glattem versbau; das letztere gilt besonders vom Judas; die form ist hier knapp, während St. Katherine mit senkungen etwas tberladen ist.

Robert von Gloucester's chronik', das hauptdenkmal in unserem metrum, zeigt in seinem versbau eine so grosse ubereinstimmung mit den stddlichen legenden, dass Marsh ohne bedenken alle legenden dem Robert of Gloacester selbst znschrieb. Horstmann will ihm zwar nicht alle legenden zuerteilen, denn er nimmt der verschiedenheit der legenden entsprechend verschiedene dichter an, aber auch or vermutet, dass Robert sich unter der zahl der dichter unserer legendensammlung befunden habe; dass diese vielleicht in der abtei von Gloucester, wo Robert mönch war, ihren ursprung hat. Sioher ist; dass Robert die legenden kannte und fur seine chronik benutzte, so. den Thomas Becket. Umgekehrt, sagt Horstmann, benntzten die dichter der legendensammlung die chronik, so z: b., fur die geographische einleitung in Kenelm. Ein endgiltiges urteil ther das verhaltniss Robert's zu der legendensammlang ist noch nicht gewonnen.

Fur unsere metrische untersuchung benutzte ich die beiden abschnitte ans der chronik bei. Wulker, Altengl. Lesebuch und die beiden in Matzner's Sprachproben.

Nach Schipper haben wir hier widerum eine mischnng von septenaren and alexandrinern. Indess ist wie in den heiligenleben die siebentreffige langzeile der grundtypus und die verse, welche anscheinend nur 6 hebungen haben, sind entweder mit schwebender betonung zu lesen oder als sehlecht gebaute oder verdorbene siebentreffige $\mathrm{zu}$ betrachten. Sie unterscheiden sich

, 1 Herausgegeben von Thomas Hearne in 2 bänden, Oxfort 1724. 
wesentlich von den alexandrinern nach französischem muster dadurch, dass das fehlen der senkung zwischen zwei wörtern oder zwei silben desselben wortes noch gestattet ist.

Im allgemeinen sind die rerse der chronik weniger glatt, als die der mehrzahl der legendenhandschriften, und die zahl der unvollkommenen verse, der sogenannten alexandriner, ist daher eine grössere. Gleich das erste sttick bei Wulker (im ganzen 190 zeilen) zeigt sehr holprige verse. Ueherall begegnen wir fehlen von senkungen und schwebender betonung. Besonders erfordern zusammengesetzte wörter dieselbe fast immer; z. b.: Soupsex (63), Estsex (63), Norbfolc, Sopfolc, Scotlonde (: Yrlonde 32), Wiltshire (65), Barkshire, Hamptshire (64), dann erchbishop (86). Ferner andere eigennamen mit schwerer zweiter silbe: Statford, Herford, Dorsete, Cordoil, Duram, London. Der auftakt feblt fast in der hälfte der verse zu anfang oder nach der cäsur.

Noch weniger glatt ist das zweite sttick bei Wulker (300 zeilen). Häufiges fehlen des auftaktes oder von senkungen im innern, mehrfacher, sogar dreisilbiger auftakt und mehrfache senkung . stören meist den regelmässigen gang des metrums. Die zahl der ganz regelrechten verse, wie z. b. v. 62:

ist sehr gering.

he zef hem ziftes gret ynow and dude us corteysie,

Schwebende betonung zeigen die wörter Hengist $(1,11)$, Gloucestre (281), soupward (56), typing (57).

Etwas besser ist das erste stlick bei Mätzner (238 zeilen). Die zahl der verse, die nur 6 hebungen zeigen, ist geringer; es gehören dazu verse wie $2,3,6,92,174,197,220$. Allo ubrigen lassen sich ohne muthe mit 7 hebungen lesen, auch ohne dass man 'schlechte septenare aus guten sechshebern macht'. Allerdings sind dann wider viele falle schwebender betonung anzunehmen; aber ohne dieses zugeständniss lassen sich viele verse auch nicht als alexandriner lesen, und wenn Schipper betonungen wie Nórpwéy, Bápúlf, úpwárd, dónwárd, kýndóm, trénnésse, gódnésse, éndýng, áskýng, die durch den reim gesichert sind, zugibt, warum sollen wir nicht auch ausserhalb des reimes so betonen? z. b. Leicéstre, woduroh v. 4 eine regelrechte siebentreffige langzeile. wird, wómmón (193), Hennin, Mórgín (192), frénschipe (142), kýndóm. Auch Rosenthal's betonung fairést am schlusse von v. 8 mag richtig sein, 
obgleich im innern des verses die superlativendung gewöhnlich keinen zweiten treff erhalt.

Ebenso zeigt das zweite stuck bei Matzner (279 zeilen) nur selten verse von nur 6 hebungen. Betonungen wie Nórbwéy (: pley 30), hórdóm (: kinedom 244), drýnýnge (: synge 149), fálshéde (104), Bástárd, Háráld, Wýllám sind teilweise durch den reim gesichert.

Trautmann nimmt fur Robert's zeit sogar noch treffigungen wie kníztés, batćilé an, also ganz wie es frther regel war. Indess sind diese fälle sehr unsicher, ansserdem sehr selten und nur in den weniger regelmässig gebauten ersten halbversen zu finden, so dass wir wol eher mangelnde hebung zuzugeben haben. Die zweiten halbverse wenigstens erfordern nie diese treffigung, sondern zeigen anch ohne sie drei hebungen.

Metrisch nahe verwant mit den legenden und Robert's chronik ist ein bruchsttlck aus dem ms. Harl. 2277, von Wright in seinen Popular treatises on Science written during the Middle-Ages in Anglo-Saxon, Anglo-Norman, and English, London 1841, betitelt: Fragment on Popular Science. Anch in stil und sprache schliesst es sich an jene denkmäler an. Der vers ist im wesentlichen genau derselbe, den wir dort fanden. Auch hier zeigen manche verse nur 6 hebungen statt 7, wenn auch selten, z. b. v. $30,233,319$ und 354; in den letzten beiden wurden wir 7 hebungen haben bei schwebender betonung von legges und after. Letztere tritt gew 8 hnlich nur bei schwereren endsilben ein: reýnfórst (232), wómmán (294), lizling (: lhing 146), bygynnýnge (: springe 331), vellinge (: waxinge 333 ), ebenso wáxinge (335) im innern des verses, grénninge (: starynge 390).

Der auftakt am anfang fehlt fast in der hälfte der verse, nach der căsur etwas seltener. Mehrfacher auftakt. selbst dreisilbiger, sowie mehrfache senkung sind häufig.

$\mathrm{Zu}$ den denkmalern in paarweise gereimten siebentreffigen langzeilen gehoren ferner: King Salomon's Book of Wisdom und St. Jeremie's 15 Tokens before Doomday with Lamentatio Animarum.1 Einige male stehen statt 7 nur 6

1 Beide aus ms. Laud. 622 (ende des 14. jahrhunderts), herausgegeben von Furnivall, E. E. T. S. 1872. 
hebungen, indem der erste halbvers nur 3 hebungen enthält; die von Furnivall angefuhrten, darunter v. 183, 202, sind nicht die einzigen. Bisweilen tritt auch das umgekehrte verhältniss in den beiden halbversen ein, indem der erste zwar nur 3, dafür aber der zweite halbvers 4 hebungen zeigt. Doppelter, sowie fehlender auftakt, silbenverschleifung in der senkung sind noch immer häufig; auch fehlen der senkung zwischen zwei wörtern findet sich öfters, schwebende betonung dagegen selten; nur wörter wie womman, wisdom lassen diese noch zu.

In strophenform findet sich unsere langzeile bereits seit dem 13. jahrhundert verwendet, wird aber bald durch die romanischen rhythmen verdrängt. Das älteste derartige gedicht ditrte ein Pater noster sein. ${ }^{\prime}$ Es besteht ans 8 versen in der reimstellung aabbbce. Nur der zweite vers zeigt sechs hebungen, alle anderen sieben. - Fónding trägt noch zwei hebungen, alle wörter mit leichter endsilbe nur eine.

Ebenfalls aus dem 13. jahrhundert sind die beiden gedichte Doomsday und Death. ${ }^{2}$ Doomsday besteht ans 11 strophen von je 4 unter einander reimenden langzeilen. Die reime sind weiblich mit ausnahme der vierten strophe. Die ersten halbverse enden männlich oder weiblich. Die meisten verse zeigen nur 6 hebungen, aber auch regelrechte siebentreffige langzeilen sind vertreten. Auftakt und senkungen fehlen bisweilen. Schwebende betonung zeigen die wörter dai-réd, ofspring, sunfule, berninde, laverd.

Death hat 33 strophen von 4 reimenden langzeilen. Die meisten strophen zeigen weiblichen reim, nur 11 männlichen. Wider ist die zahl der regelmilssigen verse eine geringe. Auftakt und senkungen können fehlen. Sohwebende betonung verlangen die wörter cleý-clót, godspelle, ondsweare, hordom, tipinge.

Vorherrschend in regelrechten langzeilen von 7 hebungen ist geschrieben A Prayer to our Lady. ${ }^{3}$ Hier sind 44 verse zu 11 strophen von je 4 vereinigt; die vier verse einer strophe

1 Aus ms. Cambr. publ. libr. Hh VI, 11; gedruckt bei Wright und Halliwell I, 169.

2 Beide in den mss. Cott. Calig. A IX und Jes. Coll. 29; gedruckt bei Morris, An Old Engl. Miscellany s. 163 und 168.

3 Aus ms. Adddit. 2i,909; gedruckt bei Morris, \&. \&. 0. s. 192. 

Friars and their Miracle Plays ${ }^{1}$, bestehend aus 7 strophen von je 6 zeilen (aaabb). Ferner: Ballad of a tyrannical husband ${ }^{2}$, in strophen von 4 zeilen (aaaa). Hier uberwiegen die 'alexandriner' und nur wenige siebentreffige verse finden sich zerstreut darunter.

V.

Die langzeile in den übersetzungen von Fleming, Phaer, Golding und Chapman.

Eine grosse rolle spielt der septenar in der englischen literatur des 16. jahrhunderts. Wir finden ihn mit vorliebe in den ubersetzungen aus dem Lateinischen und Griechischen verwendet.

Im jahre 1575 veröffentlichte Ahraham Fleming eine ubersetzung der Bucolica Virgil's unter dem titel: The Bukolikes of Publins Virgilius Maro with alphabetical annotations etc. Drawne out into plaine and familiar Englishe, verse for verse, by Abraham Fleming, Student etc. Eine probe aus der zehnten eclogue steht in Warton's History of English poetry, edited by Hazlitt, band IV. Es sind zwar verse von 7 hebungen, aber sehr holprig gebaute. Besonders ist die cäsur sehr ungeschickt behandelt. Manche verse zeigen keine cäsur, andere schon nach der dritten hebung. Besser ist der versban in der 1589 erschienenen neuen version mit dem titel: The Bucolics of $P$. Virgilins Maro etc. otherwise called his pastorals or Shepherds Meetings; Together with his Georgies or Ruralls etc. All newly translated into English verse by A.F. Proben daraus, auf die ich mich leider beschränken muss, da mir keine ausgabe zugänglich war, finden sich ebenfalls bei Warton a. a. o. und bei Guest, History of English Rhythms. Die cäsur ist hier richtig gesetzt, nach der vierten hebung, aber in den senkungen, die mit den hebungen regelmässig wechseln, sind viele verschleifungen und elisionen nötig. Ganz schwere silben, einsilbige hauptwörter etc. finden sich in der senkung und umgekehrt ganz tonlose wörter in der hebung. Mit recht sagt Guest, sein versbau sei ebenso schlecht wie seine poesie.

Die verse sind reimlos. -

1 Ms. Cott. Clop. B II ; gedruckt bei Wright und Halliwell I, 322.

2 Bei Wright und Halliwell II, 196. 
Der zweite Virgilubersetzer dieser zeit ist $\mathrm{Pha}$ er. Seine thersetzung der ersten neun bucher und eines teiles des zehnten buches von Virgil's Aeneis wurde nach seinem tode von William Wightman veröffentlicht. Thomas Twyne, ein arzt aus Lewes, fuhrte dann das werk zu ende. Eine ausgabe von 1573 unter dem titel: The whole XII Bookes of the Aeneidos of Virgill whereof the first IX and part of the tenth were converted into English meeter by Thomas Phaer Esquier and the residue supplied and the whole worke together newly set forth by Thomas Twyne, Gentleman, befindet sich im Britischen Museum, und ist die von mir benutzte.

Der versbau ist ganz der moderne. Hebung und senkung wechseln regelmässig und nur selten entfallen auf die letztere zwei leichte silben. Besonders ist es die endung -en, die gern verschliffen wixd in heaven, given, comen; $\mathrm{z}$. b.: and given thee léave oder the sonne was comen about; ferner die endungen -er, -ey oder $-y$, wie: sister of Jbve, mother of Goddes, the countrey of cloúdy skýes, in méry arráy. Die endung -ed. der verben wird bald verschliffen, bald zählt sie als silbe; verschliffen wird sie z. b. im ersten buche in esteemed, zählt aber in flamed, astoined. Auffallend ist der vers:

they wondred at the presents there, they wondred at Jule (: rule) wo das zweite wondred zwei treffe tragen mtlsste, wenn der vers korrekt sein soll.

Bisweilen finden wir eine tonumsetzung, indem die endung statt der stammsilbe den treff erhält. So verlangt in dem bei Warton abgedruckten sttlcke das metrum threytinde. Derartige nnregelmässigkeiten sind durchaus nicht auffallend. Bei Orm sahen wir einen ausgiebigen gebrauch davon gemacht und erwahnten bei der gelegenheit, dass anch Chancer sich solche freiheiten erlaubt. Dort bezog es sich hauptsăchlich auf endungen wie -dom, -hood, -ship, -ness,--ess, -and. Später wechselt der treff besonders in zusammensetzungen. Guest in seiner History of Engl. Rhythms spricht ansfthrlich ther diesen gebrauch im Englischen. Ganz ăhnliche falle von tonumsetzung finden wir auch im Mittelhochdentschen, und anch hierin erkennen wir wider eine parallele entwickelung der deutschen und englischen metrik. Bekannt und besonders hänfig ist diese eigenttlmlichkeit im Mittelhochdentschen bei eigennamen wie Isỏll, Kudrin. Wörter mit un- haben den treff bald anf der ersten, bald auf 
der zweiten silbe, besonders dreisilbige: unmáre, seltener bei ur-: urloübes; hierher gehören auch wörter auf -liche: etliche, sumliche; ferner antwürten, herbérgen; besonders oft findet sich ellénde, schwerer ist der fall vliegénde. Zweisilbige wörter mit tonumsetzung sind: imbiss, niemán, alsó; noch auffallender swelchiu, kunnét, undé, die letzteren aber nur am versanfange und nach der cäsur (nach Wilmans). Bei Golding und Chapman werden wir einen ausgiebigeren und dem Mittelhochdeutschen ganz entsprechenden gebrauch von dieser freiheit gemacht sehen.

Im gegensatz zu Fleming's reimlosen versen sind bei Phaer je zwei durch endreim gebunden. Die reime sind sehr rein. Aeusserst selten sind weibliche reime bei Phaer wie bei Twyne.

Bisweilen findet eine verschiebung der cäsur statt, so dass die verse in verhältniss von $3: 4$ gebaut sind statt $4: 3$, d. h. die cäsur nach der dritten hebung steht. So v. 3 bei Guest:

By moving more she bredes $\|$ oder v. 5: and as she ronnes her might doth rise

With pride on ground she goth $\|$ and percith cloudes with head on hight.

Oft folgen mehrere derartig gebaute verse auf einander:

The morning next $\|$ whan first the dawing stars expulsed were

And cleare the day began \| Aeneas out from every where

Assembled al his mates $\|$ and into counsel did them call.

Than from a bank on hie \|| be thus pronounced them all.

Es findet sich auch die cäsur nach der funften hebung:

And glad at last on their acquainted shore their ships arrive.

Einige verse sind unvollständig, so v. 9 bei Warton:

In armour girt she calls.

Noch grösser sind die unregelmässigkeiten in der behandlung der cäsur bei Twyne. Nehmen wir als beispiel fur seine versification ans dem XII. buche die verse:

With daunted force and fight $\|$ unlucky late $\|$ when quaild to bee

His Latines stout of yore $\|$ most valiant Turnus prince did see, 
Himself a marke to ech mans eies \| outragious gan to boyle

And rise in rage $\|$ much like a Lyon fierce $\|$ of Affricke soyle

Whose breast when as with dint \| of hunters speare $\|$ is wounded deepe,

He then prepares himself to fight $\|$ and curled lockes doth kepe

Erect with rage upon his neck $\|$ a stickinge launce with pawes

of hunters bold he breakes || and fominge frettes with bloddie iawes:

Nonother wise the rage \| of furious Turnus forth doth breake.

Ovid fand einen ubersetzer in Golding, der seine version unter dem titel veröffentlichte: The XV Bookes of P. Ovidius Naso entituled Metamorphosis. A worke very pleasant and delectable translated out of Latin into English meeter by Arthur Golding gentleman, London 1587. Die ausgabe ist im Britischen Museum. Proben stehen wider bei Warton und Guest. Warton hält Golding für einen besseren ubersetzer und dichter als Phaer. Sein stil ist dichterisch und schwungvoll. Sein versbau ist im ganzen regelmässig. In der senkung stehen bisweilen zwei leichtere silben, z. b. die beiden letzten von gathering, Yvorie; besonders gern wird wider -en verschliffen in heaven, heavenlie, even; andere fälle von verschleifung sind violence, continuall im III. buche. Die endung -es zählt nur nach sibilanten als besondere silbe; zweisilbig sind daher: tresses, ages, scourges, einsilbig dagegen: armes, leaves, tongues, waves, (she) shakes. - Sehr verschieden und regellos ist die behandlung der verbalformen auf -ed. Zweisilbig bezw. dreisilbig gebraucht sind im I. buche: clottred, seemed, placed, nailed, lived, enclosed, confused, transformed; in den folgenden btichern: called, thanked, speckled, lurked, wrapped, wexed, forged, used, beguiled, availed, denied, replied; aus den proben bei Warton und Guest: loked, formed. Dagegen ist das $e$ der endung elidiert oder muss elidiert werden in: transformed, appeared, borrowed; marveld, accompanid, delivered, quoathd, represst; venymd, dangled. Schon die verschiedene schreibung verrät das schwanken. Selbst am versende tritt eine verschiedene schreibung ein; neben provoked : revoked (I. buch), prooved : mooved (IX. buch) finden sich mainleind : unconsireind (I. bnch), longd : prolongd (IX, buch). - 
Zweisilbig bezw. dreisilbig gebraucht sind die präsensformen auf -eth: looketh, leapeth, sheweth, beareth, permitteth. Schwerfällig sind die betonungen: cúriouslý, leisurel'y, contínualý, órderlý (: skie), décentlý (: on hie), indifferentlý, inwardlie, hártilý, hórriblie, slóuthfullý; ferner capácitie, púnishmént, oder gar trávellíng, périshélh, Ísyclés, Cárbunclés. Auch diesem analoges finden wir in der deutschen metrik; ich erwähne aus Kudrun die betonungen: rúoretén, Hártmuotés, bännierén.

Eine umsetzung des treffs findet sich in wörtern wie likewise, foresight, straitnáy, righlstránge, alwaý, bietet aber nach dem oben gesagten nichts auffälliges; always, und die anderen adverbia mit all wurden besonders gern so gebraucht, wenn auch jetzt der ton auf dem all liegt. Die treffigungen likewise und foresight finden sich in demselben verse, wo zugleich eine verschleifung im auftakt steht und seemed zweisilbig gebraucht ist (I. buch):

Even so likewise through great foresight \| to God it seemed best.

Die cäsur steht immer nach der vierten hebung. Ein beispiel fehlenden auftaktes nach der cäsur bietet sich in:

But that appeared in the heaven starres \| glistring all about.

Gegen das ende der übersetzung mehren sich die fälle weiblicher reime, die anfangs selten sind; ich rechne dahin reime wie: other: molher:brother, falher: rather, matter: water, allogither: hither: thilher, flower: power, travell:gravell, cherries : berries, readie: headie u. a. Ebenso mehren sich gegen schluss des werkes die fälle von enjambement; ein beispiel dafur aus dem VII. buche ist:

but shee

did seeme with better shere to die II for that hir conscience was.

Wie im enjambement das versende seinen einfluss auf die syntaktische konstruktion verliert, ebenso verliert auch die cäsur ihre bedeutung und fällt nicht selten zwischen grammatisch eng zusammengehöriges.

Eine Iliastbersetzung wurde bereits 1581 von Arthur Hall versucht nach einer französischen version von M. Hugues Salel (1555). Vollständig wurde sie indess erst von Chapman in's Englische ubertragen 1611: The Ilias of Homer, prince of poets, never before in any language traly translated with a comment 
on some of his chief places done according to the Greek by George Chapman. - Neuherausgegeben wurde sie London 1858 von Richard Hooper. - 1614 erschien Chapman's thersetzung der Odyssee; auch diese wurde 1857 von Rich. Hooper herausgegeben unter dem titel: The Odysseys of Homer translated according to the Greek by George Chapman. - Coleridge in seinem briefe an Wordsworth bezeichnete Chapman's Odyssee als die bessere der beiden thersetzungen. Da sie in zehnsilblern geschrieben ist, haben wir uns hier nicht näher damit za beschäftigen.

Die verse der Ilias sind weniger regelmässig als die Golding's. Hallam sagt: 'The verse is rhymed of 14 syllables, which corresponds better to the hexameter than the decasyllable couplet; he is often uncouth and often low, but the spirited and rapid flow of the metre makes him respectable to lovers of poetry'.

Ben Jonson hielt die Homertbersetzang fur prosa wegen des 'interlacing of the verses or the running of the lines into one another, so that the sense does not close with the couplet' (vgl. Blackwood's Magazine 1831-32). - Warton beschuldigt Chapman 'of labouring with the inconvenience of an awkward, inharmonious and unheroic measure, imposed by custom but disgustful to modern ears'. Warton sagt auch: 'the whole performance betrays the negligence of haste'.

Am anffallendsten ist die behandlung der cäsur. Sie hat bei Chapmann gar nicht die bedentung einer pause; ohne rtlcksicht daranf läuft die syntaktische konstruktion dahin. Oft schliesst der satz vor der cäsur, während andererseits die cäsur zwischen eng zusammengehöriges fallt. Ebenso eilt die satzkonstruktion ther das versende hinweg. Als beispiel fur. beides moggen die anfangsverse dienen:

Achilles' baneful wrath resound \|

o goddess; that imposed

Infinite sorrows on the Greeks \||

and many brave souls los'd

From breasts heroic; sent them far \|

to that invisible cave

That no light comforts; and their limbs |

to dogs and vulture gave.

Ferner aus buch XXIV v. 675 ff.:

0 Hector, all my brothers more \|

were not so loved of me 
As thy most virtues. Not my lord $\|$

I held so dear as thee

That brought me hither; before which \|

I wish I had been brought

To ruin, for what breeds that wish \|

which is the mischief wrought

By my access, yet never found one harsh taunt, one word's ill,

From thy sweet carriage.

In der probe bei Guest wird die konjunktion when durch die cäsur von dem satze abgetrennt, das beiwort ablest von Achilles. In den oben angefuhrten versen ist es besonders die trennnng des objektes vom verbum, bei voranstellung des ersteren. In der stellung der cäsur ist keine abweichung zu beobachten; sie steht streng nach der vierten hebung.

Die reime sind im allgemeinen rein. Weibliche reime sind mir nicht begegnet; wörter wie flower und power gelten als einsilbig and reimen mit our, hour. Da wo präteritalformen auf -ed im reime stehen, tritt entweder elision ein: los'd: impos'd, profess'd : best, disgrac'd : fast, embrac'd : waste, impelled : withheld, shin'd : behind, fir'd : desir'd, oder die endung erhält einen treff: sécondéd: Dioméd, distributéd: shed, bririéd: head; diese letztere betonung tritt auch bei wörtern auf -ant, -ent, -ly, -y ein: váliänt : vaunt, sufficiént : tent, instantlý : eye, hưmanly : déstiný, libertý : buy.

Im innern des verses tritt bei der verbalendung -ed fast immer elision ein: lov'd, vex'd, embrac'd, occasion'd, reverenc'd, unvalu'd; als ausnahmen nenne ich unperformed und das adject. wretched. Auch sonst neigt Chapman zu elisionen. Die einsilbig gebrauchten flower und power warden schon erwähnt; dazu kommen deflow'r, flon'ry, tow'r, iron, ferner mit elision ling'ring, offerings, seekest, sogar being. Daneben finden sich treffigungen wie émperý, sùddenlý, térriblý, lázinéss, wándering, álteréth. Heaven wird wider besonders gern einsilbig gebraucht, ferner given; im XV. buche stehen beide im reim zu einander. Andere verschleifungen sind énemies, violent, ridiculous, nátural mit nur einem treff.

Fälle von tonumsetzung in zweisilbigen wörtern sind selten. Im I. buche finden sich sicknéss, Calchás, Chrysés, dotárd, vielleicht auch rattléd in $\mathbf{v} .46$. Nicht selten dagegen stehen tonlose wörter in der hebung, schwere silben in der 
126 MENTHEL, DER OTTRWDISCHI VERS IM RNGLISCHEN.

senkung. In dem sttick bei Guest ist einmal der artikel betont in: But mightiest Fate and thé great God, das personalpronomen in: Patroclus yé left dead in field, das demonstrativpronomen in: for this dayes fast, das adjectivum: As if his áv'd steeds. In den anfangsversen findet sich das adjectivum in der hebung in: bráve souls, das possessiv-pronomen: from hér lov'd countrey; obeing his high will, die präposition: given bý Apollo.

ELBERFELD.

E. Menthel. 